$\begin{array}{cc}\text { Programa de Pós-Graduação em Engenharia de Produção - PPGEP } & \\ \text { Laboratório de Qualidade de Vida - LaQVida } & \\ \text { Universidade Tecnológica Federal do Paraná - UTFPR } & \text { REVISTA BRASILEIRA DE } \\ \text { Ponta Grossa - PR - Brasil } & \text { QUALIDADE DE VIDA }\end{array}$

\title{
Variabilidade da frequência cardíaca e desempenho de AVDs de idosos treinados e sedentários
}

\section{Heart rate variability and performance of ADLs in elderly trained and sedentary}

\author{
Lidiane França \\ Faculdade de Educação Física de Santos - FEFIS- Santos - São Paulo - Brasil \\ lidifran@hotmail.com \\ Tairine Sanches \\ Faculdade de Educação Física de Santos - FEFIS-Santos - São Paulo - Brasil \\ tairine_sanches@hotmail.com \\ Tania Regina \\ Faculdade de Educação Física de Santos - FEFIS-Santos - São Paulo - Brasil \\ taniadrenalina@yahoo.com.br \\ Fabricio Madureira \\ Faculdade de Educação Física de Santos - Santos - São Paulo - Brasil \\ shark_jaws@hotmail.com \\ Felipe Bartolotto \\ Faculdade de Educação Física de Santos - Santos - São Paulo - Brasil \\ felipe_augusto.2wf@hotmail.com \\ Emilson Colantonio \\ Universidade Federal de São Paulo - UNIFESP - Santos - São Paulo - Brasil \\ nunoec@uol.com.br \\ Claudio Scorcine \\ Faculdade de Educação Física de Santos - Santos - São Paulo - Brasil \\ claudio-scorcine@uol.com.br
}

\section{RESUMO}

OBJETIVO: Avaliar grupos de idosos que praticam musculação, hidroginástica e sedentários no desempenho de atividades de vida diária (AVDs) e índices de variabilidade da frequência cardíaca (VFC).

MÉTODOS: Foram avaliados 60 idosos separados em três grupos distintos (G1 - praticantes de musculação; G2 - praticantes de hidroginástica; G3 - sedentários). Foi avaliada a VFC em repouso durante cinco minutos antes dos exercícios propostos. Testes físicos propostos: $800 \mathrm{~m}$ de corrida ou caminhada, levantar da cadeira e se locomover, levantar do solo, subir e descer 15 degraus e calçar meias.

RESULTADOS: No desempenho de AVDs, o G1 apresentou resultados superiores aos demais grupos para o teste de $800 \mathrm{~m}$ de caminhada, para os testes de subir e descer escadas, levantar da cadeira e se deslocar e levantar do solo; o G1 apresentou resultados superiores ao G3 para o teste de calçar 
meias. Para as comparações entre o G2 e G3 não foi possível observar diferenças significativas. Para os índices de VFC não foi possível observar diferenças significantes entre os grupos.

CONCLUSÃO: O grupo praticante de musculação demonstrou resultados superiores na maior parte dos testes quando comparado ao grupo sedentário. Para os testes de VFC não foram encontradas diferenças significantes para nenhum dos grupos.

PALAVRAS-CHAVE: Atividades de vida diária. Frequência cardíaca. Idosos.

\begin{abstract}
OBJECTIVE: To evaluate groups of elderly people who practice bodybuilding, aerobics and sedentary performance of ADLs and HRV indices.

METHODS: We evaluated 60 elderly separated into three distinct groups G1 that performs bodybuilding training, aerobics and sedentary G2 G3. HRV was evaluated at rest for five minutes before the proposed exercises. Proposed physical tests: $800 \mathrm{~m}$ running or walking, rising from a chair and moving around, lifting the ground, up and down 15 steps and put on socks.

RESULTS: In performing ADLs G1 showed superior results to other groups for the $800 \mathrm{~m}$ walk test, for tests up and down stairs, getting up from the chair and move around and get up soil G1 showed superior results the G3, for putting on socks and test for comparisons between the G2 and G3 did not exhibit significant differences. To the HRV indices did not exhibit significant differences between the groups.
\end{abstract}

CONCLUSIONS: The weight training group showed superior results in most tests when compared to the sedentary group, for tests of HRV not significant for either group.

KEYWORDS: Activities of daily living. Heart rate. Seniors.

\title{
1. Introdução
}

O envelhecimento é a perda progressiva das aptidões funcionais do organismo dos seres vivos, podendo potencializar o aumento do sedentarismo (KALLINEN; MARKKU, 1995). Segundo Andreotti e Okuma (1999), aproximadamente $25 \%$ da população mundial idosa depende de outras pessoas para realizar suas tarefas cotidianas. Entretanto, a grande maioria, apesar de apresentar alguma dificuldade na realização dessas atividades, é independente. Esses dados estão diretamente ligados as principais modificações que ocorrem com o envelhecimento, como a perda de eficiência do aparelho motor com a diminuição de força e potência, alterações morfológicas e bioquímicas (IMAMURA; IMAMURA; HIROSE-PASTOR, 1999).

Alterações estruturais e funcionais também ocorrem no sistema cardíaco. Essas alterações influenciam com uma menor resposta do sistema simpato-vagal, diminuindo as respostas das vias simpáticas e parassimpáticas e, consequentemente, a variabilidade da frequência cardíaca (VFC) no idoso (UMETANI et al., 1998; LAKATTA, 2002). Essa diminuição da VFC vem sendo estudada e relacionada com diversas patologias, entre elas, com as doenças pulmonares crônicas (FURULAND et al., 2008). Outros riscos são relatados como mortalidade após infarto agudo, hipertensão arterial (MENEZES JÚNIOR; MOREIRA; DAHER, 2004), entre outras doenças degenerativas (WOLF et al., 1977; KLEIGER et al., 1987; PUMPRLA et al., 2002). Portanto, a VFC, como uma ferramenta não invasiva, torna-se um instrumento para avaliação de aplicabilidade facilitada, além de seus resultados serem de grande valia para a população estudada (ACHTEN; JEUKENDRUP, 2003).

Através da atividade física, os efeitos deletérios do envelhecimento podem ser minimizados (MATSUDO, 2002). Estudos demonstram os efeitos positivos do treinamento, através de modalidades de força como a musculação (SILVA; MATSUURA, 2002; PEDRO; BERNARDESAMORIM, 2008) e hidroginástica (TSUKAHARA et al., 1994; ALVES et al., 2004; KUWANO; 
SILVEIRA, 2002), em idosos com prática sistemática dessas modalidades. Entretanto, quando comparada a VFC entre idosos e sedentários, a literatura ainda carece de informações concretas se o treinamento seria capaz de influenciar positivamente as variáveis envolvidas.

Ainda, idosos ativos envolvidos em diferentes programas de treinamento, teria um desempenho diferenciado nas atividades de vida diária (AVDs)? Essas questões ainda são inconclusivas na literatura, o que torna a presente investigação interessante. Sendo assim o objetivo do presente estudo é analisar a VFC e o desempenho de AVDs entre idosos praticantes de musculação, hidroginástica e sedentários.

\section{Metodologia}

A amostra foi constituída por 60 idosos, separados em três grupos distintos (G1 - praticantes de musculação; G2 - praticantes de hidroginástica; G3 - sedentários).

Os testes foram realizados em um único dia, com horário pré-determinado pelos pesquisadores, através dos seguintes procedimentos: na posição sentada, os voluntários ficaram cinco minutos com o Polar RS 800 para a análise da VFC logo após a realização da bateria de testes de AVDs validadas por Andreotti e Okuma (1999), que consiste em caminhar ou correr por 800m, sentar e se deslocar por uma distância pequena pré-determinada, levantar-se do solo, subir e descer 15 degraus e calçar meias. Todos os testes foram explicados e demonstrados por profissionais de Educação Física antes de serem realizados. Após o término da bateria de testes, os idosos ficaram mais três minutos em repouso sentados para a aferição da VFC.

Os dados da VFC foram analisados através do uso do programa Kubios 2.0, verificando o comportamento da frequência cardíaca e sua variabilidade.

Para a análise da normalidade dos dados optou-se pelo teste de Shapiro Wilk e após a não confirmação, optou-se por utilizar o teste de Mann Whitney U para a comparação dos grupos estudados.

\section{Resultados}

Os valores médios e desvio padrão dos testes de AVDs e suas respectivas tarefas para os três grupos de sujeitos podem ser observados na Tabela 1:

Tabela 1 - Resultados dos testes de AVDs em segundos para 800m de caminhada; calçar meias; subir escada; descer escada; levantar-se da cadeira e se deslocar e levantar do solo

\begin{tabular}{c|cccccc}
\hline Grupo & $800 \mathrm{~m}(\mathrm{seg})$ & Calçar meias & Subir & Descer & Cadeira & Levantar \\
\hline Musculação & $470,0 \pm 130,3^{*}$ & $13,4 \pm 8,5$ & $7,0 \pm 2,4^{\wedge}$ & $6,9 \pm 2,2^{\wedge}$ & $33,1 \pm 8,2^{\wedge}$ & $3,4 \pm 1,6^{\wedge}$ \\
Hidroginástica & $608,5 \pm 132,1$ & $12,8 \pm 4,9$ & $7,8 \pm 1,8$ & $7,6 \pm 2,1$ & $30,9 \pm 11,8$ & $6,4 \pm 6,9$ \\
Sedentário & $603,2 \pm 90,9$ & $12,7 \pm 5,1$ & $9,0 \pm 2,6$ & $9,1 \pm 2,9$ & $40,5 \pm 9,2$ & $4,7 \pm 1,5$ \\
\hline
\end{tabular}

O nível de significância foi aceito em $\mathrm{P}=0,05$ e * indica diferenças significativas entre os grupos musculação e hidroginástica, ${ }^{\wedge}$ indica diferenças significativas entre os grupos musculação e sedentários.

Fonte: Autoria própria (2013).

Da mesma forma, a Tabela 2 mostra os valores médios e desvio padrão das variáveis associadas à VFC da amostra. Sendo essas RRs média dos intervalos das ondas RR; SDNN desvio padrão da média dos RR; BPM média dos batimentos por minuto; RMSSD a média dos valores absolutos das diferenças sucessivas; LF/ms índice que indica a ativação do sistema simpático; HF/ms índice de ativação do sistema parassimpático. LF/HF interação entre os dois sistemas. 
Tabela 2 - Resultados em forma de média e desvio padrão das variáveis de VFC; RR; SDNN; BPM; RMSSD; LF/ms; $\mathrm{HF} / \mathrm{ms} ; \mathrm{LF} / \mathrm{HF}$

\begin{tabular}{c|ccccccc}
\hline Grupo & RR & SDNN & BPM & RMSSD & LF/MS & HF/ms & LF/HF \\
\hline G1 & $756,7 \pm 224,0$ & $47,1 \pm 59,8$ & $93,2 \pm 58,3$ & $21,1 \pm 21,8$ & $517,2 \pm 652,7$ & $289,0 \pm 618,1$ & $5,1 \pm 3,7$ \\
G2 & $763,0 \pm 120,8$ & $30,9 \pm 6,2$ & $80,5 \pm 12,7$ & $16,9 \pm 11,9$ & $260,5 \pm 247,5$ & $98,1 \pm 127,4$ & $3,6 \pm 1,5$ \\
G3 & $772,6 \pm 58,1$ & $38,6 \pm 14,4$ & $78,3 \pm 6,2$ & $28,8 \pm 21,1$ & $500,5 \pm 419,0$ & $404,3 \pm 563,1$ & $3,4 \pm 2,6$ \\
\hline
\end{tabular}

$\mathrm{O}$ nível de significância foi aceito em $\mathrm{P} \leq 0,05 \mathrm{e} *$ indica diferença significativa entre os grupos musculação e

hidroginástica; ${ }^{\wedge}$ indica diferença entre os grupos musculação e sedentário.

Fonte: Autoria própria (2013).

\section{Discussão}

Para o teste de 800m, o G1 apresentou resultados superiores aos demais grupos: 138,5 segundos a menos que o grupo hidroginástica e 133,2 segundos para o grupo sedentário. Para os testes subir escadas, descer escadas, levantar-se da cadeira, e levantar-se do solo o G1 apresentou resultados superiores ao G3. Para o teste de calçar meias, não foram observadas diferenças significativas. Esses dados corroboram com parte da literatura acadêmica, demonstrando que a atividade física parece ser importante para que o idoso consiga manter a qualidade de vida, realizando suas AVDs sem a necessidade de auxílio por parte de outras pessoas (BORGES; MOREIRA, 2009; GUIMARÃES et al., 2008).

Sobre a variável VFC, não houve diferença significativa entre os grupos estudados, demonstrando que o fator idade parece ser determinante para a diminuição da atuação parassimpática nessa população como os achados de Nascimento et al. (2013), que avaliou a VFC em diferentes faixas etárias encontrando diferenças significativas, demonstrando uma diminuição dos índices de VFC para a população acima de 51 anos (PASCHOAL et al., 2006). Evidenciando que a idade predomina sobre o treinamento, um estudo com 22 indivíduos de meia idade constatou que, após três meses de treinamento, não houve modificações na VFC (LOPES et al., 2007).

Para os testes de AVDs, os achados do presente estudo corroboraram com o estudo de Borges e Moreira (2009) que concluiu que idosos praticantes de treinamento de força possuem desempenho superior aos idosos sedentários nas AVDs.

Em outro estudo que investigou os benefícios do treinamento de força em 70 idosos, foi observado que a atividade física é indispensável para a manutenção da qualidade de vida (GUIMARÃES et al., 2008). Os achados do presente estudo corroboram parcialmente com a literatura, demonstrando que idosos envolvidos em treinamento de força apresentam resultados superiores a idosos sedentários como no estudo de Pedro e Bernardes-Amorim (2008), que analisaram 16 indivíduos idosos, sendo 8 praticantes de musculação e 8 não praticantes, e constataram que o treinamento de força pode ser efetivo no equilíbrio de indivíduos idosos, podendo auxiliá-los na realização de AVDs.

Em outro estudo, foram investigados 60 indivíduos, variando entre 65 e 75 anos de idade, em condições de pré e pós-programa de treinamento. Foi observado que os efeitos da prática sistemática de exercícios físicos sobre índices de queda e tendência às mesmas, bem como a minimização de efeitos deletérios do envelhecimento sobre a saúde mental, são positivos e vão além dos benefícios físicos investigados no presente estudo (ACHTEN; JEUKENDRUP, 2003).

Ainda, em outro estudo, foi analisada a evolução do perfil neuromotor e capacidade funcional de mulheres ativas no período de um ano, de acordo com a idade cronológica. Foi constatado que em um ano não houve nenhuma alteração no desempenho neuromotor. Enquanto que a velocidade de levantar da cadeira e a velocidade de andar apresentaram diferenças significativas nos grupos de 5059 e 60-69 anos, com resultados entre 10 e $20 \%$ melhores. Para o teste velocidade máxima de andar, houve melhora de $8 \%$ nos grupos de 60 a 79 anos, demonstrando os benefícios da atividade física para a população idosa (MATSUDO et al., 2003).

Quanto ao grupo de praticantes de hidroginástica, os achados do presente estudo corroboram parcialmente com o estudo de Araújo e Barbosa (2007), o qual não encontrou diferenças significativas 
para a maioria dos testes em relação ao grupo praticante de musculação. Entretanto, os resultados encontrados contrariam a maior parte dos trabalhos documentados na literatura, quando não foram encontradas diferenças significativas para o grupo hidroginástica em relação ao grupo sedentário. No estudo de Kuwano e Silveira (2002), com 40 indivíduos do sexo feminino, dos quais 20 praticavam hidroginástica e 20 eram sedentários, constatou-se que os indivíduos praticantes de hidroginástica obtiveram resultados melhores nos aspectos físicos, psíquico e social. Da mesma forma, Alves et al. (2004) demonstraram que a prática da hidroginástica, em 37 mulheres idosas, foi eficiente para a melhora da aptidão física.

Programas de treinamento de força e hidroginástica contribuem para melhora na execução das AVDs, como mostrou o estudo de Passos et al. (2008) que observou diferenças significativas para os sujeitos que praticaram hidroginástica, com melhoras nos testes de sentar e levantar da cadeira em $13,5 \%$, subir degraus em $14,8 \%$, subir escadas em $8,8 \%$, levantar-se do solo em $22,8 \%$ e calçar meias em $26,3 \%$. Uma limitação do presente estudo é o fato de que as comparações foram realizadas obedecendo a um desenho experimental transversal. Portanto, não é possível afirmar que os melhores resultados alcançados pelo G1 estão diretamente associados ao efeito do treinamento. Entretanto, diversos estudos demonstraram benefícios da prática desta modalidade para a população de idosos.

\section{Considerações finais}

Os idosos que realizaram o treinamento de musculação apresentaram resultados superiores do que o grupo de sedentários para os testes de subir escadas, descer escadas, levantar-se e se deslocar, e levantar-se do solo. Para o teste de $800 \mathrm{~m}$ de caminhada ou corrida, o grupo que praticou musculação foi superior aos demais grupos estudados. Esses achados corroboram com outros estudos que avaliaram idosos treinados e sedentários. Para os testes de VFC não foi possível observar diferenças significativas entre os grupos, demonstrando que o fator idade pode ser determinante para a diminuição da VFC. Entretanto, mais estudos devem ser conduzidos concentrando-se no efeito do treinamento e com um número maior de avaliações em momentos distintos.

\section{Referências}

ACHTEN, J.; JEUKENDRUP, A. E. Heart rate monitoring. Sports Medicine, v. 33, n. 7, p. 517-538, 2003. crossef

ALVES, R. V.; MOTA, J.; COSTA, M. da C.; ALVES, J. G. B. Aptidão física relacionada à saúde de idosos: influência da hidroginástica. Revista Brasileira de Medicina do Esporte, v. 10, n. 1, p. 31-37, jan./fev. 2004. crossef

ANDREOTTI, R. A.; OKUMA, S. S. Validação de uma bateria de testes de atividades da vida diária para idosos fisicamente independentes. Revista Paulista de Educação Física, v. 13, n. 1, p. 46-66, jan./jun. 1999.

ARAÚJO, R. de C. M.; BARBOSA, M. T. da S. Análise comparativa da força muscular de mulheres idosas praticantes de ginástica e hidroginástica. Movimentum - Revista Digital de Educação Física, Ipatinga, v. 2, n. 1, p. 1-10, fev./jul. 2007.

BORGES, M. R. D.; MOREIRA, A. K. Influências da prática de atividades físicas na terceira idade: estudo comparativo dos níveis de autonomia para o desempenho nas AVDs e AIVDs entre idosos ativos fisicamente e idosos sedentários. Motriz, Rio Claro, v. 15, n. 3, p. 562-573, jul./set. 2009. 
FURULAND, H.; LINDE, T.; ENGLUND, A.; WIKSTRÖM, B. Heart rate variability is decreased in chronic kidney disease but may improve with hemoglobin normalization. Journal Nephrology, v. 21, n. 1, p. 45-52, Jan./Feb. 2008.

GUIMARÃES, A. C.; ROCHA; C. A. Q. C.; GOMES, A. L. M.; CADER, S. A.; DANTAS, E. H. M. Efeitos de um programa de atividade física sobre o nível de autonomia de idosos participantes do programa de saúde da família. Fitness \& Performance Journal, n. 1, p. 5-9, 2008.

IMAMURA, S. T.; IMAMURA, M.; HIROSE-PASTOR, E. Efeitos do envelhecimento e do exercício físico sobre o colágeno do músculo esquelético humano. Revista Brasileira de Reumatologia, v. 39, n. 1, p. 35-40, jan./fev. 1999.

KALLINEN, M.; MARKKU, A. Aging, physical activity and sports injuries. Sports Medicine, v. 20, n. 1, p. 41-52, July 1995. Crossef

KLEIGER, R. E.; MILLER, J. P.; BIGGER JR., J. T.; MOSS, A. J. Decreased heart rate variability and its association with increased mortality after acute myocardial infarction. The American Journal of Cardiology, v. 59, n. 4, p. 256-262, Feb. 1987. Crossef

KUWANO, V. G.; SILVEIRA, A. M. da. A influência da atividade física sistematizada na autopercepção do idoso em relação às atividades da vida diária. Revista da Educação Física/UEM, Maringá, v. 13, n. 2, p. 35-39, 2002.

LAKATTA, E. G. Age-associated cardiovascular changes in health: impact on cardiovascular disease in older persons. Heart Failure Reviews, v. 7, n. 1, p. 29-49, Jan. 2002. Crossef

LOPES, F. L.; PEREIRA, F. M.; REBOREDO, M. M.; CASTRO, T. M.; VIANNA, J. M.; NOVO JR., J. M.; SILVA, L. P.. Redução da variabilidade da frequiência cardíaca em indivíduos de meiaidade e o efeito do treinamento de força. Revista Brasileira de Fisioterapia, v. 11, n. 2, p. 113-119, 2007. crossef

MATSUDO, S. M. Envelhecimento, atividade física e saúde. Revista Mineira de Educação Física, Viçosa, v. 10, n. 1, p. 193-207, 2002.

MATSUDO, S. M.; MATSUDO, V. K. R.; BARROS NETO, T. L. de; ARAÚJO, T. L. de. Evolução do perfil neuromotor e capacidade funcional de mulheres fisicamente ativas de acordo com a idade cronológica. Revista Brasileira de Medicina do Esporte, v. 9, n. 6, p. 365-376, nov./dez. 2003. crossef

MENEZES JÚNIOR, A. da S.; MOREIRA, H. G.; DAHER, M. T. Análise da variabilidade da freqüência cardíaca em pacientes hipertensos, antes e depois do tratamento com inibidores da enzima conversora da angiotensina II. Arquivos Brasileiros de Cardiologia, v. 83, n. 2, p. 165-168, ago. 2004.

NASCIMENTO, L. S. do; SANTOS, A. da C.; ANDRADE LIMA, A. H. R. de; RITTI-DIAS, R. M.; BRASILEIRO-SANTOS, M. do S. Comparação da análise simbólica da variabilidade da frequência cardíaca entre mulheres fisicamente ativas de meia-idade e idosas. Revista Brasileira de Atividade Física \& Saúde, v. 18, n. 2, p. 253-259, mar. 2013. rossef

PASCHOAL, M. A.; VOLANTI, V. M.; PIRES, C. S.; FERNANDES, F. C.. Variabilidade da frequiência cardíaca em diferentes faixas etárias. Revista Brasileira de Fisioterapia, v. 10, n. 4, p. 413-419, out./dez. 2006. rossef 
PASSOS, B. M. A.; SOUZA, L. H. R.; SILVA, F. M. da; LIMA, R. M.; OLIVEIRA, R. J. de. Contribuições da hidroginástica nas atividades da vida diária e na flexibilidade de mulheres idosas. Revista da Educação Física/UEM, Maringá, v. 19, n. 1, p. 71-76, 2008.

PEDRO, E. M.; BERNARDES-AMORIM, D. Análise comparativa da massa e força muscular e do equilíbrio entre indivíduos idosos praticantes e não praticantes de musculação. Conexões: Revista da Faculdade de Educação Física da UNICAMP, Campinas, v. 6, ed. especial, p. 174-183, jul. 2008.

PUMPRLA, J.; HOWORKA, K.; GROVES, D.; CHESTER, M.; NOLAN, J. Functional assessment of heart rate variability: physiological basis and practical applications. International Journal of Cardiology, v. 84, n. 1, p. 1-14, July 2002. rossef

SILVA, V. F. da; MATSUURA, C. Efeitos da prática regular de atividade física sobre o estado cognitivo ea prevenção de quedas em idosos. Fitness \& Performance Journal, v. 1, n. 3, p. 39-45, 2002.

TSUKAHARA, N.; TODA, A.; GOTO, J.; EZAWA, I. Cross-sectional and longitudinal studies on the effect of water exercise in controlling bone loss in Japanese postmenopausal women. Journal of Nutritional Science and Vitaminology, v. 40, n. 1, p. 37-47, 1994. crossef

UMETANI, K.; SINGER, D. H.; MCCRATY, R.; ATKINSON, M. Twenty-four hour time domain heart rate variability and heart rate: relations to age and gender over nine decades. Journal of the American College of Cardiology, v. 31, n. 3, p. 593-601, Mar. 1998. Crossef

WOLF, N. M.; SANTAMORE, W.: KREULEN, T. H., BOVE, A. A.; SPANN, J. F. A heart model for understanding three-deminsional cardiac anatomy. Catheterization and Cardiovascular Diagnosis, v. 3, n. 2, p. 183-186, 1977. Crossef 